\title{
ELEMENTS OF MAXIMAL ORDER IN FINITE $p$-GROUPS
}

\author{
GUY T. HOGAN
}

\begin{abstract}
Let $G$ be a finite $p$-group such that every 3-generator subgroup has class at most $p$. If $K(G)$ denotes the subgroup of $G$ generated by the elements of maximal order in $G$, then $K(G)$ has index at most $p$ in $G$.
\end{abstract}

G. Zappa proved [5] that if $G$ is a finite $p$-group of class at most $p$, then $H_{p}(G)$ has index at most $p$ in $G$, unless $H_{p}(G)=1$. Here $H_{p}(G)$ denotes the subgroup of $G$ generated by those elements of $G$ having order different from $p$. If we let $K(G)$ be the subgroup of $G$ generated by the elements of maximal order in $G$, then, of course, $K(G) \subseteq H_{p}(G)$, whenever $H_{p}(G) \neq 1$. So we describe a construction which yields a family of examples, for each odd prime $p$ and arbitrary exponent greater than $p^{2}$, of finite $p$-groups of class $p$ with $K(G) \neq H_{p}(G)$. Thus, our result is a strengthening of Zappa's, although the techniques are essentially the same. Recently, I. D. Macdonald [3], [4] extended Zappa's results in a different direction.

Our main goal is the proof of the following

THEOREM. Let $G$ be a finite p-group such that every 3-generator subgroup has class at most $p$. Then $[G: K(G)] \leqq p$.

The notation will be the same as in [1]. The computational aspect of the proof will be facilitated greatly by the following lemma.

Lemma. Let $G$ be a finite p-group of exponent $p^{m+1}$, with the exponent of $G_{2}$ a divisor of $p^{m}$. If $y \in K(G)$, and $x \in G \backslash K(G)$, then

$$
y^{-p^{m}} \equiv\left[y,{ }_{p-1} x\right]^{p^{m-1}} \quad\left(\bmod G_{p+1}\right) .
$$

Proof. Since we argue modulo $G_{p+1}$, we may as well assume that $G_{p+1}=1$, that is, $G$ has class at most $p$. From $y \in K(G)$, and $x \in G \backslash K(G)$, it follows that $x y \in G \backslash K(G)$. By the Hall-Petrescu Identity [2, Chapter III, Satz 9.4, Hilfsatz 9.5],

$$
x^{p^{m}} y^{p^{m}}=(x y)^{p^{m}} \prod_{k=2}^{p^{m}} C_{k}^{\left(\begin{array}{c}
p^{m} \\
k
\end{array}\right)}
$$

Presented to the Society, March 27, 1970; received by the editors May 7, 1971. AMS 1970 subject classifications. Primary 20D15, $20 \mathrm{D} 25$.

Key words and phrases. Finite $p$-group, maximal order, exponent, $H_{p}$-subgroup.

(c) American Mathematical Society 1972 
where $C_{k} \in G_{k}$, and $C_{p}=\left[y,{ }_{p-1} x\right]^{a} \prod_{i} V_{i}^{a_{i}}, a \equiv-1(\bmod p)$, and each $V_{i}$ has the form $\left[y, x, z_{2}, \cdots, z_{p-2}\right]$ with each $z_{j}$ equal to $x$ or $y$, and at least one equal to $y$. But $p^{m}$ divides $\left(\begin{array}{c}p^{m} \\ k\end{array}\right)$ if $k<p$, and, by assumption, $c(G) \leqq p$ and $\exp \left(G_{2}\right)$ divides $p^{m}$. Thus, (1) reduces to

$$
\left.y^{p^{m}}=C_{p}^{\left(\begin{array}{c}
p^{m} \\
p
\end{array}\right)}=\left\{\left[y,{ }_{p-1} x\right]^{a} \prod_{i} V_{i}^{a_{i}}\right\}^{\left(^{m}{ }_{p}\right.}\right) \text {. }
$$

This may be further simplified if we observe that $\left(\begin{array}{c}p^{m} \\ p\end{array}\right) \equiv p^{m-1}\left(\bmod p^{m}\right)$, so that we may replace $\left(\begin{array}{c}p^{m} \\ p\end{array}\right)$, in (2), by $p^{m-1}$. Now let $W_{k}$ be the product of all the $V_{i}^{a_{i}}$ 's which have weight $k$ in $x$, and weight $p-k$ in $y(k=1,2, \cdots$, $p-2$ ), and observe that (2) remains valid if we replace $x$ by $x^{r}, 1 \leqq r \leqq p-1$. We then get, for each of these $r$,

$$
y^{p^{m}}=\left\{\left[y,{ }_{p-1} x^{r}\right]^{a} \prod_{k=1}^{p-2} W_{k}^{r^{k}}\right\}^{p^{m-1}} .
$$

If we now multiply these $p-1$ quantities together, ignoring the ordering of the factors since they all belong to $Z(G)$, we get

$$
y^{p^{m}(p-1)}=\left\{\left[y,{ }_{p-1} x\right]^{a} \sum_{r=1}^{p-1} r^{p-1} \prod_{k=1}^{p-2} W_{k} \sum_{r=1}^{p-1} r^{k}\right\}^{p^{m-1}},
$$

or, on further reduction,

$$
y^{-p^{m}}=\left[y,{ }_{p-1} x\right]^{a p^{m-1}} \sum_{r=1}^{p-1} r^{p-1} \prod_{k=1}^{p-2} W_{k}^{p^{m-1}} \sum_{r=1}^{p-1} r^{k} .
$$

But $a \equiv-1(\bmod p)$, and $\sum_{r=1}^{p-1} r^{p-1} \equiv-1(\bmod p)$. Furthermore, $\sum_{r=1}^{p-1} r^{k} \equiv 0$ $(\bmod p)$, if $1 \leqq k \leqq p-2$. Since the exponent of $G_{2}$ divides $p^{m}$, we see that the powers of $W_{k}$ above are all equal to the identity, so that finally we have $y^{-p^{m}}=\left[y,{ }_{p-1} x\right]^{p^{m-1}}$ which proves the lemma.

We are now ready to prove the theorem.

Proof. Assume the theorem false and let $G$ be a minimal counterexample, so that $[G: K(G)] \geqq p^{2}$. Then there exists a subgroup $V \subseteq G$, such that $K(G) \subset V \subseteq G$, and $[V: K(G)]=p^{2}$. Clearly, then, $K(V)=K(G)$, and $V$ satisfies the hypothesis of the theorem. By minimality of the order of $G$, this gives $V=G$, so $K(G)$ has index $p^{2}$ in $G$. Pick elements $a, b$ in $G \backslash K(G)$ such that $G=\langle a, b, K(G)\rangle$ and let $y$ be an element of order $p^{m+1}=$ exponent of $G$. If $U=\langle a, b, y\rangle$, then $\exp (U)=\exp (G)$, so that $K(U) \subseteq K(G)$. Hence, $K(U) \subseteq U \cap K(G)$. Since $G=U K(G)$, then if $U$ were a proper subgroup of $G$, we would have

$$
p \geqq[U: K(U)] \geqq[U: U \cap K(G)]=[U K(G): K(G)]=[G: K(G)]=p^{2} .
$$

Thus, $G=U=\langle a, b, y\rangle$, so that $G$ is a 3-generator group, and $G$ has class $p$.

With a view toward applying the lemma, we now show that $G_{2}$ has exponent dividing $p^{m}$. Let $L=\langle a, d\rangle$, where $d \in G_{2}$. Then $L_{2}$ is generated by 
$[a, d]$ and all its conjugates in $L$, so that $L_{2} \subseteq G_{3}$. Then $L_{3} \subseteq G_{4}$, and, continuing thus, we have $L_{p} \subseteq G_{p+1}=1$. This means that $L$ has class at most $p-1$. Hence, $L$ is a regular $p$-group, so that $L=K(L)$. If $d$ had order $p^{m+1}$, then $L=K(L) \subseteq K(G)$. But this cannot occur since $a \in L \backslash K(G)$. Therefore, $\exp \left(G_{2}\right)<\exp (G)$.

Now suppose we could find $x \in G \backslash K(G)$ such that $x^{p} \notin K(G)$. Let $x^{p}=u$. By the lemma, we have

$$
\begin{aligned}
y^{-p^{m}} & =\left[y,{ }_{p-1} u\right]^{p^{m-1}}=\left[y,{ }_{p-2} u, x^{p}\right]^{p^{m-1}} \\
& =\left[y,{ }_{p-2} u, x\right]^{p^{m}}=1
\end{aligned}
$$

since the commutators above belong to $Z(G) \cap G_{2}$. But this contradicts the fact that $\exp (G)=p^{m+1}$. We may assume, therefore, that $a$ and $b$ are so chosen that $a K(G)$ and $b K(G)$ are independent generators of $G / K(G)$. Then $a^{r} b^{s} \in K(G)$ iff $r \equiv s \equiv 0(\bmod p)$. So, by the lemma,

$$
y^{-p^{m}}=\left[y,{ }_{p-1} a^{r} b\right]^{p^{m-1}}
$$

where we are taking $r=1,2, \cdots, p-1$. Expanding on the right, recalling that $G$ has class $p$, we get

$$
y^{-p^{m}}=\left\{\prod_{k=0}^{p-1} W_{k}^{r^{k}}\right\}^{p^{m-1}}
$$

where $W_{k}$ is the product of all commutators having the form

$$
\left[y, z_{1}, z_{2}, \cdots z_{p-1}\right],
$$

and each $z_{j}=a$ or $b$, with $k$ of them equal to $a$, the remaining $p-k-1$, of course, equal to $b$. In particular, $W_{0}=\left[y,{ }_{p-1} b\right]$ and $W_{p-1}=\left[y,_{p-1} a\right]$. Isolating these two factors, and using the congruence $r^{p-1} \equiv 1(\bmod p)$, if $r \not \equiv 0(\bmod p)$, we get

$$
y^{-p^{m}}=W_{0}^{p^{m-1}} W_{p-1}^{p^{m-1}} \prod_{k=1}^{p-2} W_{k}^{p^{m-1} r^{k}} .
$$

Now we multiply these equations together, for $r=1,2, \cdots, p-1$ making use of the relation $\sum_{r=1}^{p-1} r^{k} \equiv 0(\bmod p)$, if $1 \leqq k \leqq p-2$, and the fact that the exponent of $G_{p}$ divides $p^{m}$. This gives

$$
y^{p^{m}}=W_{0}^{-p^{m-1}} W_{p-1}^{-p^{m-1}} .
$$

But, by the lemma, $y^{p^{m}}=W_{0}^{-p^{m-1}}$. This forces $W_{p-1}^{p^{m-1}}=1$, so that, again, by the lemma, $y^{p^{m}}=1$. This contradiction proves the theorem.

EXAMPLE. We construct a family of examples, for each odd prime $p$ and exponent $p^{r+1}, r \geqq 2$, of finite $p$-groups, $G$, of class $p$, with $[G: K(G)]=p$, and $H_{p}(G)=G$. 
Let $p$ be any odd prime, and $r>1$ be an integer. Let

$$
A=\left\langle a_{1}\right\rangle \times\left\langle a_{2}\right\rangle \times \cdots \times\left\langle a_{p-1}\right\rangle \times\langle b\rangle
$$

with $\left|a_{1}\right|=p^{r+1},\left|a_{k}\right|=p^{r}$, for $1<k<p$, and $|b|=p^{r-1}$. Define an automorphism, $x$, of $A$ by

$$
\begin{aligned}
a_{k}^{x} & =a_{k} a_{k+1} \text { for } 1 \leqq k<p-1, \\
a_{p-1}^{x} & =a_{p-1} a_{1}^{-p} b, \\
b^{x} & =b a_{2}^{p} .
\end{aligned}
$$

Let $G$ be the split extension of $A$ by $\langle x\rangle$. For convenience, let $a_{p}=b a_{1}^{-p}$. Then $G=\left\langle a_{1}, x\right\rangle$ is metabelian so the formulas of Lemma 3 in [1] may be used to simplify some of the computations below.

$$
\begin{aligned}
{\left[a_{1},{ }_{k} x\right] } & =\left[a_{k}, x\right]=a_{k+1}, \quad 1 \leqq k<p-1, \\
{\left[a_{1},{ }_{p-1} x\right] } & =\left[a_{p-1}, x\right]=a_{1}^{-p} b=a_{p}, \\
{\left[a_{1},{ }_{p} x\right] } & =\left[a_{p}, x\right]=[b, x]\left[a_{1}, x\right]^{-p}=a_{2}^{p} a_{2}^{-p}=1 .
\end{aligned}
$$

Thus, $G$ has class $p$, and $\exp \left(G_{2}\right)=p^{r}$. For any $a \in A$,

$$
\left[a, x^{p^{r}}\right]=\prod_{j=1}^{p^{r}}\left[a,{ }_{j} x\right]^{\left(\begin{array}{c}
p^{r} \\
j
\end{array}\right)}=\prod_{j=1}^{p-1}\left[a,{ }_{j} x\right]^{\left(\begin{array}{c}
p^{r} \\
j
\end{array}\right)}=1,
$$

and inspection of the corresponding equations for $\left[a, x^{p^{r-1}}\right]$ shows that $x$ has order $p^{r}$. Now $A$ has exponent $p^{r+1}$, and if $1 \leqq k<p^{r}, a \in A$, we find, by Lemma 3 of $[\mathbf{1}]$,

$$
\left(a x^{-k}\right)^{p^{r}}=a^{p^{r}} \prod_{0<i+j<p^{r}}\left[a,{ }_{i} x^{k},{ }_{j} a\right]^{(i+j+1}{ }^{p^{r}} x^{-k p^{r}}
$$

which simplifies to

$$
\left(a x^{-k}\right)^{p^{r}}=a^{p^{r}}\left[a,{ }_{p-1} x\right]^{k^{p-1}\left(\begin{array}{l}
p^{r} \\
p
\end{array}\right)}=a^{p^{r}}\left[a,{ }_{p-1} x\right]^{k^{p-1} p^{r-1}}
$$

since $\left(\begin{array}{c}p^{r} \\ p\end{array}\right) \equiv p^{r-1}\left(\bmod p^{r}\right)$, and $\exp \left(G_{2}\right)=p^{r}$. But the element above has order at most $p$, so that $\exp (G)=p^{r+1}$. Taking $a=a_{1}$, we see that $\left(a_{1} x^{-p}\right)^{p^{r}}=$ $a_{1}^{p^{r}} \neq 1$, so that $a_{1} x^{-p} \in K(G)$. Since $A \subseteq K(G)$, this gives $x^{-p} \in K(G)$. Hence, $A\left\langle x^{p}\right\rangle \subseteq K(G)$. The elements of $G \backslash A\left\langle x^{p}\right\rangle$ are all expressible in the form $a x^{-k}$ for $k \not \equiv 0(\bmod p)$, so for these values of $k$ we have $k^{p-1} \equiv 1(\bmod p)$. Writing $a=\prod_{i=1}^{p} a_{i}^{e_{i}}$ we have

$$
\left(a x^{-k}\right)^{p^{r}}=\left(a_{1}^{p} a_{p}\right)^{e_{1} p^{r-1}}=b^{e_{1} p^{r-1}}=1 .
$$

Hence, $K(G)=A\left\langle x^{p}\right\rangle$, and, of course, $[G: K(G)]=p$. Finally, $x \in H_{p}(G)$, and $a_{1} \in H_{p}(G)$, so $H(G)=G_{p}$. 


\section{REFERENCES}

1. G. T. Hogan and W. P. Kappe, On the $H_{p}$-problem for finite p-groups, Proc. Amer. Math. Soc. 20 (1969), 450-454. MR 39 \#312.

2. B. Huppert, Endliche Gruppen. I, Die Grundlehren der math. Wissenschaften, Band 134, Springer-Verlag, Berlin, 1967. MR 37 \#302.

3. I. D. Macdonald, The Hughes problem and others, J. Austral. Math. Soc. 10 (1969), 475-479. MR 40 \#7353.

4. - Solution of the Hughes problem for finite p-groups of class $2 p-2$, Proc. Amer. Math. Soc. 27 (1971), 39-42.

5. G. Zappa, Contributo allo studio del problema di Hughes sui gruppi, Ann. Mat. Pura Appl. (4) 57 (1962), 211-219. MR 25 \#1210.

Department of Mathematics, State University College of New York, Oneonta, NEW YORK 13820 